\title{
A Qualitative Research for Defining Meaningful Attributes for the Treatment of Inflammatory Bowel Disease from the Patient Perspective
}

\author{
Edouard Louis ${ }^{1}$. Juan M. Ramos-Goñi ${ }^{2}$. Jesus Cuervo ${ }^{2}$ - Uri Kopylov ${ }^{3,4}$ - Manuel Barreiro-de Acosta ${ }^{5}$. \\ Sara McCartney ${ }^{6}$. Greg Rosenfeld ${ }^{7}$ (D) Dominik Bettenworth ${ }^{8}$ (1) $\cdot$ Ailsa Hart ${ }^{9} \cdot$ Kerri Novak $^{10} \cdot$ Xavier Donnet $^{11}$. \\ David Easton $^{12} \cdot$ Roberto Saldaña $^{13} \cdot$ Katja Protze $^{14} \cdot$ Eyal Tzur $^{15} \cdot$ Gabriela Alperovich $^{16} \cdot$ Francesc Casellas $^{17}$
}

Published online: 30 January 2020

(c) The Author(s) 2020

\begin{abstract}
Introduction Crohn's disease (CD) and ulcerative colitis (UC) are chronic, inflammatory bowel diseases (IBD). Each class and type of medication available for the treatment of IBD has distinct characteristics and long-term effects that a patient may consider. We present the results of qualitative research that aimed to develop a descriptive framework that outlines the most relevant disease and/or treatment attributes for IBD treatment decisions and focuses on the patient perspective.

Methods This research employed a three-step approach: a literature review to identify a broad list of attributes, a focus group meeting including patients and clinicians to assess the relevance of the attributes, and two rounds of voting to name and define each attribute. The literature review was used to develop the initial list of attributes. Although the same attributes were defined for both $\mathrm{UC}$ and $\mathrm{CD}$, the relative importance of each attribute to UC or CD was considered. The list of attributes was discussed and evaluated in the focus group meeting, which included eight patient representatives and nine gastroenterologists. Using feedback elicited from the focus group meeting, the research team developed a draft of the descriptive framework that grouped the attributes into domain subsets. All members of the focus group participated in two subsequent rounds of structured, online voting, which was used to refine the wording to name and define each attribute. Additionally, participants ranked all the attributes included in the descriptive framework to suggest which attributes were less relevant and could be omitted.

Results Among 574 publications retrieved from the databases and registries, we identified 32 eligible publications, and an initial list of attributes was developed. This list was refined during the focus group meeting, resulting in a draft descriptive framework of attributes within subsets of domains. The final descriptive framework was developed based on structured rounds of online voting to further refine attribute names and definitions. In the final descriptive framework, a total of ten attributes were identified: abdominal pain, other disease-related pain, bowel urgency, fatigue, risk of cancer and serious infections within the next 10 years, risk of mild to moderate complications, aesthetic complications related to treatment, emotional status, sexual life, and social life and relationships. These attributes were distributed across three domains: efficacy, complications and risk, and health-related quality of life.

Conclusions Through the identification of the ten most relevant attributes that influence patient decision making for IBD treatments, we developed a descriptive framework that should be considered by physicians when discussing IBD treatment options with their patients. The results of our qualitative research may also be helpful for the development of future IBD clinical studies and quantitative research.
\end{abstract}

This article is part of the topical collection "Formative qualitative evidence for health preference and outcomes research".

Electronic supplementary material The online version of this article (https://doi.org/10.1007/s40271-019-00407-5) contains supplementary material, which is available to authorized users.

Extended author information available on the last page of the article

\section{Introduction}

Crohn's disease (CD) and ulcerative colitis (UC) are chronic, systemic inflammatory bowel diseases (IBD) with significant potential burden and resulting disability for patients $[1,2]$. The incidence and prevalence of UC and CD vary 


\section{Key Points}

This research identified the ten most relevant attributes within three domains that can influence patients' decisions when making treatment choices for inflammatory bowel diseases (IBD).

The descriptive framework could be used to facilitate discussion between patients and physicians when reviewing IBD treatment options.

Additional research to quantify the importance of each attribute in patient preferences is needed.

within different geographic locations. The most prevalent regions include North America, Northern Europe, and Australia, but prevalence is increasing in regions where this has historically been low, such as Africa, South America, and Asia [3, 4].

Patients experience IBD differently based on the severity of the disease, the coexistence of other autoimmune diseases or extra-intestinal manifestations; and treatment for these conditions varies according to diverse patterns of clinical practice $[5,6]$. Medications used for the treatment of IBD include aminosalicylates, glucocorticoids, immunomodulators, biologics, and small molecules, with each class having distinct characteristics that will influence patient choice. Patient and physician preferences for IBD treatment options may also differ, thus communication between patients and physicians is crucial in the shared decision-making process [7].

Qualitative patient preference data identify which outcomes, endpoints, or treatment attributes are relevant to patients, whereas quantitative patient preference data estimate how much the different attributes matter to patients and the trade-offs that patients are willing to make [8]. Although the qualitative approach required to develop descriptive frameworks to describe treatment alternatives has been thoroughly described [9], there is limited information on which qualitative data or methods should be used to conduct a quantitative study on patient preferences. In the past, descriptive frameworks for health preference research were developed based on literature, non-preference-based health surveys, or expert opinion (top-down methods) [10]. More recently, the bottom-up methodology has been prevailing over the top-down methods. In this approach, patients are considered central to the process and their experiences and opinions on how the disease affects their lives are incorporated into the methodology [8].

Without a clear understanding of the patient perspective, the results of preference studies can be inconclusive or misleading. Although there is no consensus on which qualitative methods should be applied after a literature review, there is agreement that literature searches alone are insufficient to adequately develop a descriptive framework for a robust preferences study [9]. The use of focus groups, in-depth interviews, and Delphi panels can be useful and provide a stronger methodology for qualitative research $[11,12]$.

To date, there is limited research that evaluates how different treatment characteristics may affect treatment choice for IBD patients, particularly with respect to the increasing range of mechanisms of action of new agents that will be entering the IBD space in the next few years. Moreover, the published literature on patients' values/preferences for IBD treatment presents highly variable results [13-17].

The objective of the study presented here was to create a descriptive framework that includes the most relevant attributes that influence patients' decision making regarding IBD treatment. The current research should serve as a solid foundation for future preference elicitation studies analysing the utility values of the attributes.

\section{Methods}

A literature review, a focus group meeting, and structured online voting were conducted to identify the most relevant attributes associated with treatment decision making in IBD.

\subsection{Literature Review}

A literature review was performed on published health preference studies to identify preferences or attitudes towards pharmacological treatment in adult patients suffering from mild to severe IBD conditions. To inform and facilitate the literature search, we used the Population/ Problem, Intervention, Comparison, Outcome, Study type (PICOS) framework. Both general and highly specialized international databases and registries (PubMed, Cochrane Library, International Society for Pharmacoeconomics and Outcomes Research, ClinicalTrials.gov, and the Health Preference Study and Technology Registry of the International Academy of Health Preference Research-https:// hpstr.org/landing) were searched from January 1, 2000 to June 19, 2018, using the medical subject heading (MeSH) terms "preference", "attitude to health", "willingness to pay", "time trade-off", "standard gamble", "conjoint", "discrete choice", "best-worst", "utility", "inflammatory bowel disease", "Crohn disease", and "ulcerative colitis" \{e.g. ((()((inflammatory bowel disease[Title/Abstract] OR Crohn Disease[Title/Abstract] OR ulcerative colitis)[Title/ Abstract] AND ((preference[Title/Abstract] OR attitude to health[Title/Abstract] OR willingness to pay[Title/ 
Abstract] OR time trade-off[Title/Abstract] OR discrete choice[Title/Abstract] OR conjoint[Title/Abstract] OR utility[Title/Abstract])) AND (“2000/01/01”[Date - Publication]: “2018/06/19"[Date - Publication])) AND (therapy)) NOT osteopathy) NOT surgery $\}$. Preference research of both qualitative and quantitative research as well as articles on input from patients and clinical experts were included. Publications related to surgery and alternative medical options were excluded.

Two reviewers independently screened the titles of the resulting citations for eligibility, and articles that were out of scope, duplicates, based on animal models, or studies in paediatric populations were discarded. Out of scope articles included publications focused on general satisfaction with health care provision, probiotics, preferences about being involved in shared decision making decisions, or satisfaction in the transition from paediatric to adulthood management of IBD. Publications that aimed to explore factors associated with health service utilization and preference for services, aimed to assess the appropriateness of immunosuppressive therapy in different scenarios, preferences on exercise, studies in pregnant women to evaluate their adherence, general reviews on adherence and preferences for psychological interventions, general overview/ reviews, opinion letters, overview of quality improvement initiatives in IBD, state or art of treatments and management, preferences for diagnostic procedures were also considered out of scope.

Following the title screening step, the abstracts of the remaining articles were reviewed using the same criteria. The reviewers then independently screened the full text of the remaining articles following the abstract screening using the same criteria as the first two steps. Reviewers adjudicated conflicts through discussion or consultation with a third reviewer. After the title, abstract, and full-text screening steps, the reviewers identified and classified the relevant attributes across different domains (e.g. effectiveness, side effects, health-related quality of life [HRQoL], and well-being). Generic attributes, such as reduction of side effects, were not considered in this research. Although the same attributes were defined for UC and $C D$, the relative importance of each attribute to $\mathrm{UC}$ or $\mathrm{CD}$ was considered.

\subsection{Focus Group Meeting}

We aimed to conduct a focus group of approximately 16-18 participants to determine patients' values and opinions regarding different treatment attributes. To have different perspectives, an invitation letter to participate in the research was sent to patient associations and gastroenterologists from several countries. We aimed to recruit one patient representative per gastroenterologist. Those who expressed an interest in participating were contacted directly by the research team, and, at that point, the research project was explained in more detail. A written agreement was obtained if they were aged 18 years or older, were able to speak English, and could travel to the focus group meeting venue to participate.

The focus group meeting was conducted on September 28, 2018 in Frankfurt, Germany. To determine the most relevant treatment attributes while considering both patient and physician perspectives, eight patient representatives and nine gastroenterologists were included. Patients represented local patient associations or local groups from countries such as Belgium, Canada, Spain, Germany, the UK, and Israel. The gastroenterologists were IBD specialists from academic medical centres with special interest and/or expertise in patient preferences, quality of life, and patientreported outcomes, and represented the same countries as the patients. In addition to providing their perspective on the attributes, the gastroenterologists also provided clinical expertise.

Information on the objectives, methodology, and results from the literature review was shared with all participants prior to the meeting and was further reviewed with the focus group participants during the focus group meeting. Additionally, the results of the literature review and an explanation of the identified domains and attributes were provided. Through discussion, the focus group participants aligned on which attributes should be included in each domain. All participants had the chance to state their opinions regarding the importance of each different treatment attribute.

Although the focus group meeting was not recorded, a transcript of the discussions was developed by a stenographer. Using the transcript, a tally was developed to indicate the number of participants who considered each attribute meaningful. These responses informed the subsequent draft of the descriptive framework that included the attributes grouped into domain subsets.

\subsection{Structured Rounds of Voting}

Following the focus group, the eight patient representatives and nine gastroenterologists participated in two rounds of online voting with the objective of refining the specific wording of the descriptive framework. The structured rounds of voting were designed to minimize the potential bias introduced by the research team or dominant participants in the focus group meeting by providing patients with the opportunity to individually review the information and to contribute feedback without the influence of other participants/ colleagues.

The simplified list of attributes included in the descriptive framework was shared during the first round of voting. For each attribute, a question provided suggested wording for 
that attribute and asked whether the participant agreed or not; participants also had the opportunity to suggest additional changes to the wording. Additionally, participants were asked to rank all the attributes included in the descriptive framework from most to least relevant (global ranking task). All responses from the wording questions and global ranking task were analysed using qualitative methods for free-text comments and frequencies for response options. The free-text comments were analysed to identify valuable context to better understand the responses or to improve the wording of the attributes. Based on the first round of voting, a refined version of the descriptive framework was developed.

The second round of voting aimed to determine which attributes could potentially be removed from the final descriptive framework. This method was used to minimize the final number of attributes to be similar to the maximum number of attributes that are currently tested in patient preference studies. The average number of attributes reported in the literature is six, with a minimum value of two and a maximum value of 12 [18]. While more consideration was given to the patients' responses, the gastroenterologists' responses were considered when there was not a clear consensus among the patient responses. The total number of attributes included in the final list was based on the importance of each individual attribute provided by the patient participants as well as the experience of the research team in creating a valuable descriptive framework.

\section{Results}

\subsection{Literature Review}

The question statements used to inform the PICOS framework are presented in Online Resource Table S1 (see the electronic supplementary material [ESM]). The literature search identified 574 records. The titles of these citations were screened for their relevance to the research topic, resulting in 109 articles to be further assessed for eligibility by abstract screening. The abstract screening step eliminated 72 additional records. The full text of the remaining 37 publications was evaluated based on pre-specified eligibility criteria, and 32 publications on patient preferences were included in this research (Fig. 1). The most common reasons for excluding publications were out of scope (473), duplicates (20), and studies in the paediatric population (19).

A total of 127 attributes were identified based on the 32 publications (Online Resource Table S2; see the ESM). These attributes were grouped into seven different domains (effectiveness, side-effects, HRQoL, well-being, available evidence, administration/convenience, and other).

\subsection{Focus Group Meeting}

Although not identified in the selected publications, skin cancer and herpes zoster are side effects in the treatment of IBD and a matter of concern for patients and physicians [19, 20]. Based on the focus group discussion, these attributes were added to the list.

Table 1 shows the draft of the descriptive framework that was developed based on the feedback received during the focus group meeting. The draft included 16 relevant attributes within five different domains (efficacy, complications/risk, HRQoL/well-being, administration, and cost/copayment). Pain, stool control (specifically defined by bowel urgency), rectal bleeding, endoscopic results, fatigue/loss of energy, and rapidity of action of treatment were the most valued efficacy attributes based on the focus group meeting. Among the complication and risk attributes, the participants selected the risk of life-threatening cancer and serious infections within the next 10 years, risk of mild to moderate complications, aesthetic complications related to treatment, and the need to remain on steroids as being the most relevant. HRQoL and well-being attributes, such as emotional status, sexual life, social life and relationships, and functional performance in daily activities, as well as treatment administration (mode, frequency, and place of administration) and cost of treatment in general were also valued by the participants in the focus group meeting.

\subsection{Structured Voting Rounds}

A refined version of the descriptive framework was developed after the first round of voting. The full survey used in the first round of voting is available in the ESM. The number of attributes included in the structured voting was based on the global ranking task included in the first round of voting (Table 1). Stool control, pain, and fatigue/loss of energy were considered more important than endoscopic results, rectal bleeding, treatment administration, and cost according to patient participants. The attributes that received lower ranking were removed from the attribute list. Following the first round of structured voting, the descriptive framework included 14 attributes within three different domains (efficacy, complications/risk, and HRQoL/well-being).

During the second round of voting, participants expressed which attributes they would most likely remove from the final draft of the descriptive framework (Fig. 2). Stool frequency, rapidity of treatment action, and functional performance in daily activities were the most common attributes recommended for removal and thus not included in the final list. Steroid dependency was removed from the final list because of the low ranking it received during the global ranking task of the first round of voting (12th place) as well as due to patient and physician feedback received during the second round of 
Fig. 1 Literature flow diagram. *Used as a complementary source to confirm that all the relevant endpoints in clinical investigation had been identified. ISPOR International Society for Pharmacoeconomics and Outcomes Research, $\mathrm{NIH}$ National Institutes of Health

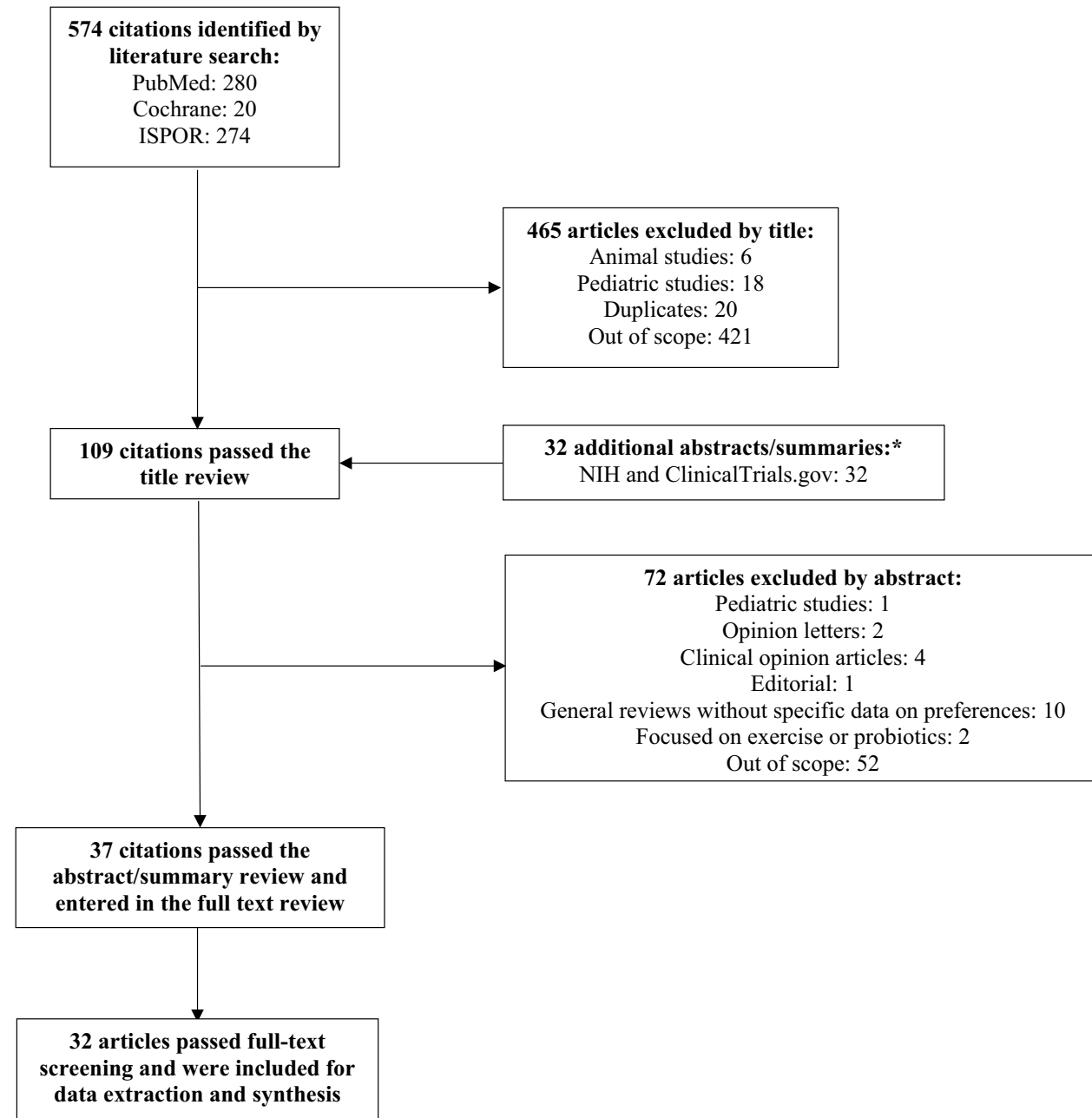

*Used as a complementary source to confirm that all the relevant endpoints in clinical investigation had been identified. voting. The full survey used in the second round of voting is available in the ESM. The final descriptive framework generated from the results of the structured surveys included ten attributes, grouped into three domains (efficacy, complications/risk, and HRQoL; Table 2).

\section{Discussion}

This study reports the process and results of a three-step approach to understand the attributes that patients most highly value when making decisions about IBD treatment. To our knowledge, this research is the first study that defines a descriptive set of attributes based on input from patients with IBD. Our research could serve as a foundation for future quantitative studies that aim at defining the importance of these attributes when making treatment decisions for IBD; the attributes presented here should be adapted to the specific needs of the study. In order to proceed to quantitative research, levels should be assigned to the selected attributes and should be tested in a patient preferences study. Results from this type of study could also be valuable in directing the evolution of endpoints used in future IBD interventional trials.

This research highlights the ten most relevant attributes across domains of efficacy, complications/risk, and HRQoL that are most influential to a patient's decision regarding IBD treatment. The resulting practical descriptive framework will enable investigators to conduct further quantitative research to better understand the priorities of patients with IBD and to estimate the relative importance of each attribute/levels by means of discrete choice methods. When these levels of importance are clear, the quantification of how much more important one attribute may be compared to another can be communicated not only to other patients, but also to other stakeholders.

Of the attributes not deemed relevant by patients during the second round of online voting, we speculate that 
Table 1 Shortened list of attributes grouped in redefined domains agreed on in the focus group meeting and patients' ranking from the first round of voting

\begin{tabular}{llr}
\hline Domain & Attribute name & Rank \\
\hline Efficacy & Pain & 2 \\
& Stool control & 1 \\
& Rectal bleeding & 14 \\
& Endoscopic results & 13 \\
& Fatigue/loss of energy & 3 \\
& Rapidity of action of treatment & 10 \\
Complications/risk & Risk of life-threatening cancer and seri- & 6 \\
& ous infections within the next 10 years & \\
& Risk of mild to moderate complications & 9 \\
& Aesthetic complications related to treat- & 8 \\
& ment & 12 \\
& Need to remain on steroids & 5 \\
HRQoL/well-being & Emotional status & 11 \\
& Sexual life & 7 \\
& Social life and relationships & 4 \\
& Functional performance in daily activi- & 4 \\
Administration & ties & 15 \\
Cost/co-payment & Treatment administration & 16 \\
\hline & Cost & \\
\hline
\end{tabular}

$H R Q o L$ health-related quality of life

"functional performance in daily activities" was excluded from the final list because patients likely associate functional performance with symptom resolution. Interestingly, stool frequency was not considered as relevant for patients as bowel urgency (defined as the need to rush to the toilet to avoid an accident), and therefore was dropped from the list. The need to remain on steroids was also deprioritized (considering the gastroenterologist perspective); nevertheless, aesthetic complications (most of them related to prolonged steroid exposure) was kept as a relevant attribute, indicating that steroid dependency is still a matter of concern.

In a recent study that used an online iterative Delphi consensus process to develop an instrument to measure IBDrelated disability, authors agreed to include ten items of high importance to both patients and physicians (abdominal pain, body image, education and work, emotions, energy, interpersonal interactions, joint pain, regulating defecation, sexual functions, and sleep) within the tool and are in agreement with the attributes identified in the current study presented here [21]. The results of the current research are also consistent with previous studies that demonstrated that patients rate quality of life higher than gains in productivity [16], and improvements in quality of life and symptom control are priority treatment objectives for IBD patients, with abdominal pain and bowel movement urgency being the most important symptoms [15].

The present research has several limitations. When used for qualitative literature review, the PICOS framework has several limitations that can impact the quality of clinical evidence obtained under its guidance [22]. Acknowledged limitations of the PICOS framework for qualitative evidence research include the fact that the framework does not accommodate terms relating to qualitative research. For example, the "Intervention" and "Comparison" components are not relevant for qualitative research, which traditionally does not utilize interventions and control groups. Additionally, combining the "Population" and "Intervention" components generally retrieves references to quantitative research. For this reason, it is possible that we were unable to capture
Fig. 2 Frequencies of stated attributes dropped by patients. *Used as a complementary source to confirm that all the relevant endpoints in clinical investigation had been identified

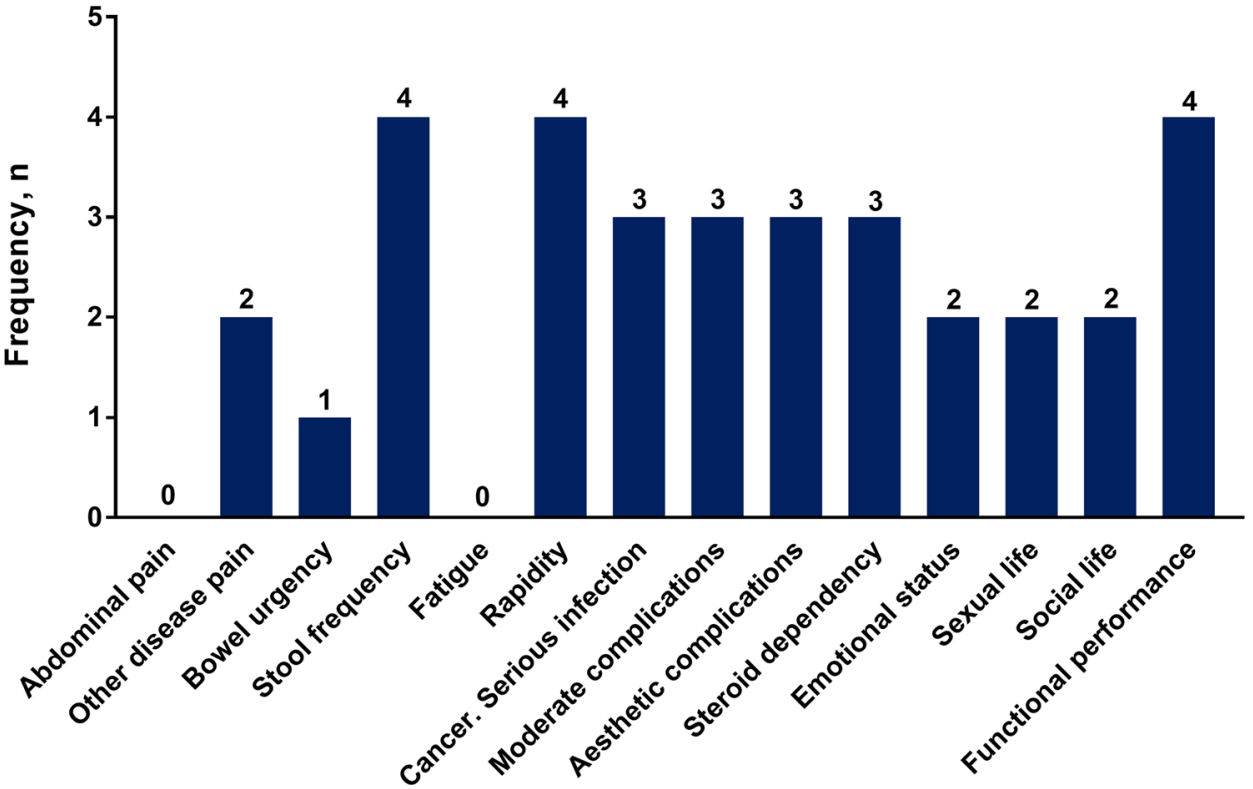


Table 2 Final list of attributes

\begin{tabular}{ll}
\hline Domain & Attribute name/descriptor \\
\hline Efficacy & Abdominal pain \\
& Other disease-related pain (anal pain, joint pain/stiffness or eye pain, etc.) \\
& Bowel urgency (when you feel the need for a bowel movement, you have to rush to the toilet to avoid an accident) \\
& Fatigue (an overwhelming sense of continuing tiredness, lack of energy or feeling exhausted that is not relieved by \\
& rest or sleep) \\
& Risk of cancer and serious infections within the next 10 years (excluding non-melanoma skin cancer) \\
& Risk of mild to moderate complications (mild/moderate complications means nausea, vomiting, headache, non- \\
Complications/risk & serious infections, lab abnormalities, skin reactions, and infusion reactions) \\
& Aesthetic complications related to treatment (hair loss, unintentional weight change, facial hair, acne, puffy face) \\
Health-related quality of life & Emotional status \\
& Sexual life \\
& Social life and relationships (interpersonal interactions) \\
\hline
\end{tabular}

and include all relevant publications. Another limitation of the literature review is that only previous health preference studies were included. By doing so, we may have missed concepts and themes from other types of studies that could have provided relevant attributes. The focus group meeting has inherent limitations that need to be acknowledged [23]. Focus groups may be influenced by one or several group members dominating the discussion and articulating their opinions alone, thus, limiting the opinions of other participants. It is also possible that the research team and its facilitation of the discussion may have influenced the participants' responses, or that the patient voice may not exhibit the same weight as the physicians. Finally, the patient participants in this study may not convey the voice of all patients with IBD, but rather the experience limited to this small group. However, the structured rounds of voting were created to minimize the potential bias introduced by more dominant participants in the focus group meeting. The focus group and the online structured voting rounds may also be limited by the small number of participants. Thus, the generalizability of the interpretation and conclusions may be affected. Finally, the same attributes were defined for UC and CD, as all the attributes included were considered meaningful for patients with both diseases. We acknowledge that the relative importance of attributes differs across diseases and should be addressed in a quantitative study.

A key strength of this research was the use of multiple approaches to identify relevant and valuable attributes in the form of literature review, focus meetings comprised of both patient and physician participants, and structured online rounds of voting to provide a broad understanding of the attributes that may influence IBD treatment decision-making. Although the opinions of both patients and physicians were taken into consideration in this research, more consideration was given to patients' responses during prioritization of the attributes, resulting in elimination of some attributes from the final list. Prioritization of the patient perspective in this study is consistent with recent statements from the National Health Council for the development of patient-centred measures, including those for the assessment of patient preferences for treatment alternatives [24]. An additional strength of this study is the broad regional representation and physicians' clinical practice patterns.

\section{Conclusions}

This research identified the ten most relevant attributes that influence patients when making treatment choices. The descriptive framework developed from this study provides a better understanding of real-world decision making for patients with IBD and should be considered by physicians and nurses when discussing treatment options with patients. The current research serves as the foundation for future studies that will evaluate the utility of these attributes within clinical practice.

Acknowledgements Ramona Vladea of AbbVie Inc. provided medical writing support in the development of this manuscript. Benjamin Craig, $\mathrm{PhD}$, Associate Professor of Economics at the University of South Florida, provided valued support in the review of the materials and input from the participants.

Author Contributions Edouard Louis, Juan M. Ramos-Goñi, Jesus Cuervo, Uri Kopylov, Manuel Barreiro-de Acosta, Sara McCartney, Greg Rosenfeld, Dominik Bettenworth, Ailsa Hart, Kerri Novak, Xavier Donnet, David Easton, Roberto Saldaña, Katja Protze, Eyal Tzur, Gabriela Alperovich, and Francesc Casellas provided critical revision of the manuscript for important intellectual content and approved the final version for submission. Edouard Louis contributed in conducting research and participating in the focus group meeting and the two rounds of voting. Juan M. Ramos-Goñi and Jesus Cuervo were part of the research group and designed the project, conducted the literature search, and planned the focus group meeting and the two rounds of voting. They also analysed the results. Uri Kopylov, Manuel 
Barreiro-de Acosta, Sara McCartney, Greg Rosenfeld, Dominik Bettenworth, Ailsa Hart, Kerri Novak, and Francesc Casellas participated as clinical experts in the focus group and in the interactive voting. Xavier Donnet, David Easton, Roberto Saldaña, Katja Protze, and Eyal Tzur participated as patient representatives in the focus group meeting and the two rounds of voting. Gabriela Alperovich proposed the research idea to the research group and provided feedback on the design of the research. She served as an observer during the focus group meeting and during the interactive rounds of voting. She also contributed to the analysis of the results.

Data Availability Statement The authors declare that the data supporting the findings of this study are available within the article and its supplementary information files.

\section{Compliance with Ethical Standards}

Funding: AbbVie Inc.

Conflict of interest: E. Louis has received consulting and/or lecture fees from AbbVie, Ferring, MSD, Falk, Takeda, Hospira, Janssen, Pfizer, and Celgene; research grants from Takeda and Pfizer; and educational grants from AbbVie, Takeda, and Janssen; and has served on advisory boards for AbbVie, Ferring, MSD, Takeda, Celgene, Hospira, and Janssen. J.M. Ramos-Goñi has received consulting fees from AbbVie for conducting this research work. However, J.M. Ramos-Goñi does not have any conflict of interest with the results of this research. J. Cuervo has received consulting fees from AbbVie for conducting this research work. However, J. Cuervo does not have any conflict of interest with respect to the results of this research. U. Kopylov has received research grants from Janssen, Takeda, and Medtronic; speaker fees from Janssen, AbbVie, Takeda, MSD, and CTS; and advisory fees from Janssen, AbbVie, and Takeda. M. Barreiro-de Acosta has received financial support from AbbVie for this research. S. McCartney has received consulting, lecturing, or advisory board fees from AbbVie, Ferring, MSD, Falk, Takeda, and Janssen. A. Hart has served as consultant, advisory board member, or speaker for AbbVie, Atlantic, Bristol-Myers Squibb, Celltrion, Falk, Ferring, Janssen, MSD, Napp Pharmaceuticals, Pfizer, Pharmacosmos, Shire, and Takeda. She also serves on the Global Steering Committee for Genentech. G. Rosenfeld has received financial support for research projects and speaking engagements, and consulting fees from several pharmaceutical companies. This support was not anticipated to impact this study. He also received support to enable him to attend the in-person meeting that formed a part of this study. K. Novak has received honoraria from AbbVie for participating in this research. D. Easton and E. Tzur have participated in AbbVie-sponsored research. R. Saldaña has not received any benefit from participating in this study. However, the patient association for which he works has received honoraria from AbbVie. $D$. Bettenworth is on the advisory board or has been a consultant for AbbVie, Amgen, Falk Foundation, Ferring, MSD, Pharmacosmos, Pfizer, Roche, Takeda, Tillotts Pharma, and Vifor. X. Donnet has received support for travel to meetings for this research study from AbbVie Inc. The non-profit patient association "Association Crohn-RCUH", for which X. Donnet volunteers (as a Crohn patient), is sponsored by AbbVie, Dr Falk, Ferring, Janssen, Janssens, Pfizer, Takeda, and UCB. $K$. Protze has received consulting fees from AbbVie for participating in this research. However, K. Protze does not have any conflict of interest with respect to the results of this research. F. Casellas has received research funding from AbbVie, MSD, Shire, Ferring, and Zambon and speaker fees from AbbVie, MSD, Shire, Ferring, Zambon, Gebro, Chiesi, and Takeda. G. Alperovich is an AbbVie employee and may own AbbVie stock and/or options.
Role of authors and sponsor The authors and AbbVie scientists designed the research and analysed and interpreted the data. AbbVie funded the research and provided writing support. All authors contributed to the development of the content. The authors and AbbVie reviewed and approved the manuscript; the authors maintained control over the final content.

Open Access This article is licensed under a Creative Commons Attribution-NonCommercial 4.0 International License, which permits any non-commercial use, sharing, adaptation, distribution and reproduction in any medium or format, as long as you give appropriate credit to the original author(s) and the source, provide a link to the Creative Commons licence, and indicate if changes were made. The images or other third party material in this article are included in the article's Creative Commons licence, unless indicated otherwise in a credit line to the material. If material is not included in the article's Creative Commons licence and your intended use is not permitted by statutory regulation or exceeds the permitted use, you will need to obtain permission directly from the copyright holder.To view a copy of this licence, visit http://creativecommons.org/licenses/by-nc/4.0/.

\section{References}

1. Baumgart DC, Sandborn WJ. Crohn's disease. Lancet. 2012;380(9853):1590-605.

2. Cosnes J, et al. Epidemiology and natural history of inflammatory bowel diseases. Gastroenterology. 2011;140(6):1785-94.

3. Kumar V, Abbas AK, Fousto N, Robbins SL, Cotran RS. Robbins and Cotran pathologic basis of disease. 9th ed. Philadelphia, PA: Saunders Elsevier; 2015.

4. Ng SC, et al. Worldwide incidence and prevalence of inflammatory bowel disease in the 21 st century: a systematic review of population-based studies. Lancet. 2018;390(10114):2769-78.

5. Conway G, et al. The impact of co-existing immune-mediated diseases on phenotype and outcomes in inflammatory bowel diseases. Aliment Pharmacol Ther. 2017;45(6):814-23.

6. Singh S, et al. Variations in the medical treatment of inflammatory bowel disease among gastroenterologists. Gastroenterol Rep (Oxf). 2018;6(1):61-4.

7. Kariburyo MF, et al. Predicting pre-emptive discussions of biologic treatment: results from an openness and preference survey of inflammatory bowel disease patients and their prescribers. Adv Ther. 2017;34(6):1398-410.

8. Stevens K, Palfreyman S. The use of qualitative methods in developing the descriptive systems of preference-based measures of health-related quality of life for use in economic evaluation. Value Health. 2012;15(8):991-8.

9. Soekhai V, et al. Methods for exploring and eliciting patient preferences in the medical product lifecycle: a literature review. Drug Discov Today. 2019;24(7):1324-31.

10. Brazier JE, et al. Developing and testing methods for deriving preference-based measures of health from condition-specific measures (and other patient-based measures of outcome). Health Technol Assess. 2012;16(32):1-114.

11. US FDA. Guidance for industry. Patient-reported outcome measures: use in medical product development to support labeling claims.2009 http://www.fda.gov/downloads/Drugs/Guidances/ UCM193282.pdf. Accessed 1 May 2019.

12. Patrick DL, et al. Content validity-establishing and reporting the evidence in newly developed patient-reported outcomes (PRO) instruments for medical product evaluation: ISPOR PRO good 
research practices task force report: part 1-eliciting concepts for a new PRO instrument. Value Health. 2011;14(8):967-77.

13. Almario CV, et al. Optimizing selection of biologics in inflammatory bowel disease: development of an online patient decision aid using conjoint analysis. Am J Gastroenterol. 2018;113(1):58-71.

14. Bewtra $M$, et al. Patient preferences for surgical versus medical therapy for ulcerative colitis. Inflamm Bowel Dis. 2014;20(1):103-14.

15. Casellas F, et al. Patient preferences for inflammatory bowel disease treatment objectives. Dig Liver Dis. 2017;49(2):152-6.

16. van Deen WK, N.D., Duran NE, Kane E, van Oijen MG, Hommes DW. Value redefined for inflammatory bowel disease patients: a choice-based conjoint analysis of patients' preferences. Quality of Life Research, 2017. 26(2): 455-465.

17. Kamp KJ, Brittain K. Factors that influence treatment and nontreatment decision making among individuals with inflammatory bowel disease: an integrative review. The Patient - Patient-Centered Outcomes Research. 2018;11(3):271-84.

18. Trapero-Bertran M, Rodriguez-Martin B, Lopez-Bastida J. What attributes should be included in a discrete choice experiment related to health technologies? A systematic literature review. PLoS ONE. 2019;14(7):e0219905.
19. Cote-Daigneault, J., et al., Herpes zoster incidence in inflammatory bowel disease patients: a population-based study. Inflamm Bowel Dis, 2018.

20. Huang SZ, et al. Risk of skin cancers in thiopurines-treated and thiopurines-untreated patients with inflammatory bowel disease: a systematic review and meta-analysis. J Gastroenterol Hepatol. 2019;34(3):507-16.

21. Ghosh S, et al. Development of the IBD Disk: a visual selfadministered tool for assessing disability in inflammatory bowel diseases. Inflamm Bowel Dis. 2017;23(3):333-40.

22. Cooke A, Smith D, Booth A. Beyond PICO: the SPIDER tool for qualitative evidence synthesis. Qual Health Res. 2012;22(10):1435-43.

23. Smithson J. Using and analysing focus groups: limitations and possibilities. Int J Soc Res Methodol. 2000;3(2):103-19.

24. National Health Council. FDA standard core clinical outcome assessments and endpoints (Notice No. NOT-FD-18-014). 2018. http://www.nationalhealthcouncil.org/sites/default/files/NHC\%20 Core\%20Clinical\%20Outcome\%20Assessments\%20Endpoint s.pdf. Accessed 3 June 2019.

\title{
Affiliations
}

\author{
Edouard Louis ${ }^{1}$. Juan M. Ramos-Goñi ${ }^{2}$. Jesus Cuervo ${ }^{2}$. Uri Kopylov ${ }^{3,4} \cdot$ Manuel Barreiro-de Acosta $^{5}$. \\ Sara McCartney ${ }^{6}$. Greg Rosenfeld ${ }^{7}\left[\right.$ Dominik Bettenworth $^{8}$ (D) . Ailsa Hart ${ }^{9} \cdot$ Kerri Novak $^{10} \cdot$ Xavier Donnet $^{11}$. \\ David Easton $^{12} \cdot$ Roberto Saldaña $^{13} \cdot$ Katja Protze $^{14} \cdot$ Eyal Tzur $^{15} \cdot$ Gabriela Alperovich $^{16} \cdot$ Francesc Casellas $^{17}$ \\ Edouard Louis \\ edouard.louis@uliege.be \\ 1 CHU de Liège et Université de Liège, Domaine du Sart \\ Tilman, 4000 Liège, Belgium \\ 2 Axentiva Solutions, Calle el Calvario, 38107 Tacoronte, \\ Santa Cruz de Tenerife, Spain \\ 3 Sheba Medical Center, Ramat Gan, Israel \\ 4 Sackler Medical School, Tel Aviv University, Tel Aviv, Israel \\ 5 Complejo Universitario de Santiago de Compostela, \\ Santiago de Compostela, Spain \\ 6 University College London Hospitals, London, UK \\ 7 University of British Columbia, 770-1190 Hornby St., \\ Vancouver, BC V3K 3V9, Canada \\ 8 Department of Medicine B, Gastroenterology \\ 10 The University of Calgary, 3280 Hospital Drive NW, \\ Calgary, AB T2N4N1C, Canada \\ 11 Association Crohn-RCUH, 2 Rue des Argentines, \\ 6110 Montigny-le-Tilleul, Belgium \\ 12 Canada Crohn's and Colitis, Canada, 220 Stiver St., Russell, \\ ON K4R 1G9, Canada \\ 13 Confederación de Asociaciones de Enfermos de Crohn y \\ Colitis Ulcerosa de España, Madrid, Spain \\ 14 Schneebergstraße 33, 01277 Dresden, Germany \\ 15 Crohn's and Colitis Foundation of Israel, Hod \\ Hasharon Towers, 4 Hacharash St., Neve Ne'eman, \\ 4524075 Hod Hasharon, Israel \\ 16 AbbVie Inc., Av. Burgos 91, 28050 Madrid, Spain \\ 17 Crohn-Colitis Care Unit (UACC), Hospital Universitari Vall \\ d'Hebron-Pso, Vall d'Hebron 119, Barcelona 08035, Spain
} and Hepatologie, University Hospital Munster, Albert-Schweitzer-Campus 1, 48149 Münster, Germany

9 London North West Healthcare, London, UK 